\title{
Flexible Needle-Tissue Interaction Modeling With Depth-Varying Mean Parameter: Preliminary Study
}

\author{
Kai Guo Yan \\ Nanyang Technological University, Singapore \\ Tarun Podder \\ Member IEEE \\ Yan Yu \\ Thomas Jefferson University, yan.yu@jefferson.edu \\ Tien-I Liu \\ California State University, Sacramento CA \\ Christopher W.S. Cheng \\ Singapore General Hospital, Singapore \\ Follow this and additional works at: https://jdc.jefferson.edu/bodinejournal \\ Part of the Oncology Commons

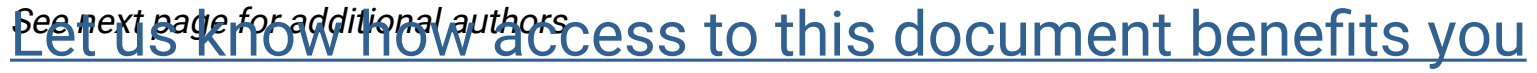

\section{Recommended Citation}

Yan, Kai Guo; Podder, Tarun; Yu, Yan; Liu, Tien-l; Cheng, Christopher W.S.; and Ng, Wan Sing (2009)

"Flexible Needle-Tissue Interaction Modeling With Depth-Varying Mean Parameter: Preliminary Study,"

Bodine Journal: Vol. 2 : Iss. 1 , Article 5.

DOI: https://doi.org/10.29046/TBJ.002.1.004

Available at: https://jdc.jefferson.edu/bodinejournal/vol2/iss1/5

This Article is brought to you for free and open access by the Jefferson Digital Commons. The Jefferson Digital Commons is a service of Thomas Jefferson University's Center for Teaching and Learning (CTL). The Commons is a showcase for Jefferson books and journals, peer-reviewed scholarly publications, unique historical collections from the University archives, and teaching tools. The Jefferson Digital Commons allows researchers and interested readers anywhere in the world to learn about and keep up to date with Jefferson scholarship. This article has been accepted for inclusion in Bodine Journal by an authorized administrator of the Jefferson Digital Commons. For more information, please contact: JeffersonDigitalCommons@jefferson.edu. 
Flexible Needle-Tissue Interaction Modeling With Depth-Varying Mean Parameter: Preliminary Study

\section{Authors}

Kai Guo Yan, Tarun Podder, Yan Yu, Tien-I Liu, Christopher W.S. Cheng, and Wan Sing Ng 


\section{Flexible Needle-Tissue Interaction Modeling With Depth-Varying Mean Parameter: Preliminary Study}

\author{
Kai Guo Yan, ${ }^{1}$ Tarun Podder, Member, IEEE, ${ }^{2}$ Yan Yu, ${ }^{2}$ Tien-I. Liu, ${ }^{3}$ \\ Christopher W. S. Cheng, ${ }^{4}$ and Wan Sing Ng, Member, IEEE ${ }^{5}$ \\ ${ }^{1}$ Nanyang Technological University, Singapore; ${ }^{2}$ Thomas Jefferson University, Philadelphia, PA, \\ USA; ${ }^{3}$ California State University, Sacramento, CA, USA; ${ }^{4}$ Singapore General Hospital, Singapore; \\ ${ }^{5}$ Nanyang Technological University, Singapore \\ (C) 2009 IEEE. Reprinted, with permission, from IEEE Trans Biomed. Eng., "Flexible needle-tissue \\ interaction modeling with depth-varying mean parameter: preliminary study", 2009 Feb;56(2):255-62.
}

\begin{abstract}
Flexible needle steering has aroused a lot of research interest in recent years. It has the potential to correct targeting errors, which may be caused by needle bending, tissue deformation, or error in insertion angle. In addition, control and planning based on a steering model can guide the needle to some areas that are currently not amenable to needles because of obstacles, such as bone or sensitive tissues. Thus, there is a clear motivation for needle steering. In this paper, a spring-beamdamper model is proposed to describe the dynamics during the needle-tissue contact procedure. Considering tissue inhomogeneity, depth-varying mean parameters are proposed to calculate the spring and damper effects. Local polynomial approximations in finite depth segments are adopted to estimate the unknown depth-varying mean parameters. Based on this approach, an online parameter estimator has been designed using the modified least-square method with a forgetting factor. Some preliminary experiments have been carried out to verify the steering model with the online parameter estimator. The details are given in this paper. Finally, conclusions and future studies are given at the end.
\end{abstract} Index Terms: Depth-varying mean parameter, needle steering modeling,
percutaneous surgery, spring-beam-damper model.

\section{Background}

Medical procedures, such as brachytherapy, biopsies, and treatment injections, require inserting a needle to a specific target location inside the human body to implant a radioactive seed, extract a tissue sample, or inject a drug. Precise needle placement is very important. Poor placement may cause tissue damage, misdiagnosis, poor dosimetry, and tumor seeding. Unfortunately, precise needle placement is hard to achieve in real practice. Errors caused by the target movement and needle deflection have been observed for a long time..$^{1-4}$ Yet to date, there are few effective physically based needle steering systems existing for correcting the targeting error automatically when it is observed. It is interesting to note that during clinic practice, some surgeons make use of a combination of lateral, twisting, and inserting motions of the needle under visual feedback from imaging systems, such as ultrasound, to correct the targeting errors. Surgeons accomplish this from experience, making it difficult to teach and limiting the accuracy to that of human hand/ eye coordination.

Flexible needle steering was first addressed by DiMaio et al. ${ }^{5}$ using a finite-element model. His model was later extended by other researchers to 3-D models. ${ }^{6,7}$ In the Medical Image Computing and Computer-Assisted Intervention Conference 2005, Daniel Glozman and Moshe Shoham ${ }^{8}$ presented a simplified virtual spring model for the needle insertion procedure. Modeling of a flexible needle was based on the assumption of quasistatic motion and a third-order polynomial was used to calculate the displacement of each element. Compromise had to be made between the computational efficiency and the model accuracy.

Needle steering making use of the needle bending has also been explored in the past few years. Some researchers have generated needle bending using different strategies, such as incorporating a prebent stylus inside a straight canula, ${ }^{9}$ or a telescoping double canula, where the internal canula is prebent. ${ }^{10}$ Other researchers showed that needles with bevel tips bend more than symmetric-tip needles. ${ }^{11}$ Making use of this effect, thin highly flexible bevel-tip needles using Nitinol were developed, and a nonholonomic model was built accordingly for steering flexible bevel-tip needles in rigid tissues. ${ }^{12}$ The nonholonomic model, a generalization of a 3-D bicycle model, was experimentally validated using a very stiff tissue phantom. Recent advances in nonholonomic path planning include stochastic model-based motion planning to compensate for noise bias, ${ }^{13}$ probabilistic models of dead-reckoning error in nonholonomic robots, ${ }^{14}$ a diffusionbased motion planning to search for a feasible path in full 3-D space, and motion planning under Markov motion uncertainty using dynamic programming to search for a feasible route while avoiding obstacles. ${ }^{15}$

In this paper, a needle steering model is proposed for flexible needle steering purpose. A spring-beam-damper model is adopted to model the dynamics between the lateral needlebased force and the corresponding lateral needle tip movement with consideration of the needle flexibility and tissue deformation. Considering the tissue inhomogeneity, depthvarying mean parameters are proposed to calculate the spring and damper effects. Local polynomial approximations in finite-depth segments are adopted to estimate the unknown depth-varying mean parameters. Unlike the models proposed in ${ }^{5}$ and, ${ }^{8}$ this model takes into consideration not only the viscoelastic tissue reactions but also the tissue inhomogeneity. In the literature, the spring-damper model has been adopted by many research groups in studying tissue deformation. ${ }^{16-18}$ But how the coupled interaction of the instrument and soft tissue is and how to control the instrument while in collision with such an environment have received little attention. Some researchers studied the collision of the flexible link with the environment in the application of grinding or surface turning operation. ${ }^{19,20}$ They modeled the environment as a simple spring-damper system, which was assumed to be stationary and was arbitrarily placed along the trajectory such that the beam would only make contact with it at the tip. In 


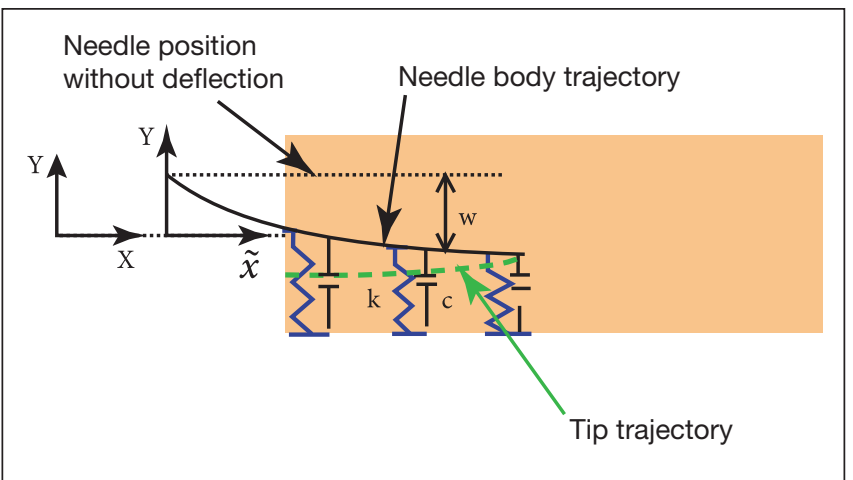

Figure 1. Mechanism of the needle insertion procedure.

the application of needle steering, the flexible instrument interacts with the environment with changing force along the needle body from time to time. The situation is much more complicated compared with the point contact.

Based on the proposed model, an online parameter estimator has been designed using the modified least-square method with a forgetting factor. Preliminary experiments have been carried out to verify the steering model with the online parameter estimator. Results have shown its effectiveness. Finally, conclusions and future studies are given at the end.

\section{Needle Lateral Steering Force Modeling And Analysis}

\section{A. Needle Lateral Steering Force Modeling}

A spring-beam-damper system, as shown in Fig. 1, is considered in this study to model the system dynamics between the lateral steering forces acting at the needle base and the corresponding needle tip lateral movement during insertion in the soft tissue. The flexible needle is assumed to follow the Bernoulli-Euler beam model and is required to be clamped tightly at the base. The initial lengths of springs are decided by the needle tip trajectory, as shown in the figure. At the beginning, the needle is placed next to the tissue. With time progressing on, the needle inserts into the tissue. Then, the springs and dampers come into contact with the needles and exert forces on it accordingly. The forces of the springs at time instant $t$ are determined by the needle body shape at that time and the needle tip trajectory; while the forces of the dampers are determined by the velocities of the contact points. During this procedure, not only the tissue deformation and the needle flexibility, but also their interaction effects, should be taken into consideration.

To derive the equations of needle insertion, the following assumptions are made.

1) For simplicity, the needle is considered to move only in the $X Y$ plane. $X$ is the insertion direction and $Y$ is the steering direction.

2) There is no longitudinal compression of the beam and only lateral deflection is possible. Furthermore, the lateral deflection of the beam is small compared with the length of the beam.

3) The rotational effect of the beam with respect to the local coordinate system is neglected.
Under the aforesaid assumptions, the system dynamic equation can be derived using Hamilton's principle as follows:

$$
\int_{t_{1}}^{t_{2}}\left(\delta T-\delta V+\delta W_{\mathrm{nc}}\right) d t=0
$$

where $T$ is the kinetic energy, $V$ is the potential energy, and $W_{\text {nc }}$ is the work done by nonconservative forces.

A local coordinate system is introduced by the Galilean transformation to replace the fixed coordinates $(x)$ with a moving coordinate system $(\tilde{x})$, which is attached at the needle base and moves with it. $v_{x}$ is the needle insertion velocity, which is assumed to be constant for modeling simplicity.

$$
\tilde{x}=x-v_{x} t \quad \text { and } \quad \tilde{y}=y .
$$

The system kinetic energy $T$ includes the kinetic energy of the fixture and the needle, as shown in the first and second terms of the following equation (3), while the potential energy $V$ includes the potential energy of the needle caused by needle bending (the first term) and the potential energy of the springs resulting from the forces between the needle and the tissue (the second term), as given in (4). The integration of the difference between the needle body positions and the needle tip trajectory gives the summation of the elongated or compressed spring length (decided by the sign of the difference) at the contact points. Because only the needle portion inside the tissue has springs exerting force on it (5), the Heaviside unit step function is used to exclude the portion outside the tissue. $h(x)=L-v_{x} t$ is the position of the insertion point in the moving coordinates system at time instant $t$. Thus, the potential energy of the springs can be calculated using the second term of (4)

$$
\begin{aligned}
& T= \frac{1}{2} M \dot{y}^{2}+\int_{0}^{L} \frac{1}{2} \rho(\dot{y}(t)+\dot{\omega}(\tilde{x}, t))^{2} d \tilde{x}=T(\dot{y}, \dot{\omega}) \\
& V= \int_{0}^{L} \frac{1}{2} E I \omega^{\prime \prime^{2}} d \tilde{x}+\int_{0}^{L} H\left(\tilde{x}-L+v_{x} t\right) \\
& \times \frac{1}{2} k\left[y(t)+\omega(\tilde{x}, t)-y\left(t+\frac{\tilde{x}-L}{v_{x}}\right)\right. \\
&\left.-\omega\left(L, \frac{\tilde{x}-L}{v_{x}}+t\right)\right]^{2} d \tilde{x} \\
& \qquad(t)=L- t \\
& \rightleftarrows \int_{0}^{L} \frac{1}{2} E I \omega^{\prime \prime^{2}} d \tilde{x}+\int_{0}^{L} H(\tilde{x}-h(t)) \\
& \times \frac{1}{2} k\left[y(t)+\omega(\tilde{x}, t)-y\left(\frac{\tilde{x}-h(t)}{v_{x}}\right)\right. \\
&\left.-\omega\left(L, \frac{\tilde{x}-h(t)}{v_{x}}\right)\right]^{2} d \tilde{x} \\
& H(x)=\left\{\begin{array}{c}
1, \\
0, \\
0
\end{array}\right] 0 . \\
& x<0
\end{aligned}
$$


Here, $M$ is the mass of the fixture that links the needle with the 3-D motion platform, $L$ is the length of the elastic beam, $\rho$ is the mass per unit length of the elastic beam, $E$ is the Young's modulus of the needle, $I$ is the second moment of inertia about the $z$-axis, $k$ is the stiffness coefficient of the spring per unit length, $c$ is the damper coefficient per unit length, $y$ is the needle base position in $y$-axis, $\dot{y}(t)$ is the corresponding velocity at time instant $t, \omega$ is the deflection of the beam along the needle body at time instance $t$, and $\dot{\omega}$ and $\omega^{\prime \prime}$ are the first and second derivatives of the beam deflection with respect to time and space, respectively.

The virtual work done by all the nonconservative forces (steering force $F_{y}$ and damping forces), is given by

$\delta W_{\mathrm{nc}}=F_{y} \delta y-\int_{0}^{L} H\left(\tilde{x}-L+v_{x} t\right) c(\dot{y}+\dot{\omega}) \delta(y+\omega) d \tilde{x}$.

The equation of motion and the boundary conditions of the system are obtained by substituting the aforesaid equations (3), (4), and (6) into (1), integrating the resulting equation by parts, and considering that the time $t 1$ and $t 2$ are arbitrary and that $\delta y, \delta \omega$ are arbitrary and independent. Thus, the equations of motion for the spring-damper system are obtained as follows:

$$
\begin{aligned}
F(y, t)= & M \ddot{y}+\int_{0}^{L} \rho(\ddot{y}+\ddot{\omega}) d \tilde{x} \\
& +\int_{0}^{L} H\left(\tilde{x}-L+v_{x} t\right)[k(y+\omega(\tilde{x}, t) \\
& \left.\left.-y\left(t_{1}\right)-\omega\left(L, t_{1}\right)\right)+c(\dot{y}+\dot{\omega}(\tilde{x}, t))\right] d \tilde{x}
\end{aligned}
$$

$$
\begin{aligned}
E I \omega^{(4)}+ & \rho(\ddot{y}+\ddot{\omega})+H\left(\tilde{x}-L+v_{x} t\right)\left[k \left(y+\omega(\tilde{x}, t)-y\left(t_{1}\right)\right.\right. \\
& \left.\left.-\omega\left(L, t_{1}\right)\right)+c(\dot{y}+\dot{\omega}(\tilde{x}, t))\right]=0
\end{aligned}
$$

where $t_{1}=t+\frac{\tilde{x}-L}{v_{x}}$.

Boundary conditions:

$$
\begin{aligned}
& \text { (i) } \omega(x, 0)=\omega(0, t)=0 \text {, (ii) } \omega^{\prime}(0, t)=0 \\
& \text { (iii) } \omega^{\prime \prime}(L, t)=\omega^{\prime \prime \prime}(L, t)=0 .
\end{aligned}
$$

To solve the partial differential equations shown in (7) and (8), unconstrained modal analysis is adopted in this approach. ${ }^{21}$ The deflection of the elastic beam and the displacement of the fixture are expressed, respectively, in terms of $\mathrm{n}$ mode shapes using the obtained $\varphi(\tilde{x}), \beta_{i}, q_{i}(t)$ as follows:

$$
\omega(\tilde{x}, t)=\sum_{i=1}^{n} \phi_{i}(\tilde{x}) q_{i}(t)=\sum_{i=1}^{n}\left[\varphi_{i}(\tilde{x})-\beta_{i}\right] q_{i}(t)
$$

and accordingly, the position of fixture is given as

$$
y(t)=\alpha(t)+\sum_{i=1}^{n} \beta_{i} q_{i}(t)
$$

where $\alpha(t)$ describes the motion of the center of mass of the total system without perturbation, $\phi(\tilde{x})$ is the shape function that is the space solution of the deflection, $q(t)$ is the time-varying amplitude of motion that is the time solution of the deflection, and $\beta$ is defined to satisfy

$$
\beta=-\frac{\rho}{M_{t}} \int_{0}^{L} \phi(\tilde{x}) d \tilde{x} .
$$

After some algebraic manipulation (refer to [22] for more details), the model is finally obtained as follows:

$$
\begin{aligned}
& \dot{\alpha}(t) \int_{L-v_{x} t}^{L} c \varphi_{i}(\tilde{x}) d \tilde{x}+\alpha(t) \int_{L-v_{x} t}^{L} k \varphi_{i}(\tilde{x}) d \tilde{x} \\
& \quad+\sum_{j}^{n} \dot{q}_{j}(t) \int_{L-v_{x} t}^{L} c \varphi_{i}(\tilde{x}) \varphi_{j}(\tilde{x}) d \tilde{x} \\
& \quad+\ddot{q}_{i}(t)+\sum_{j}^{n} q_{j}(t) \int_{L-v_{x} t}^{L} k \varphi_{i}(\tilde{x}) \varphi_{j}(\tilde{x}) d \tilde{x}+\xi_{i}^{2} q_{i}(t) \\
& =F_{y} \beta_{i}+f_{1 i} \\
& M_{t} \ddot{\alpha}(t)+c v_{x} \dot{\alpha}(t) t+\sum_{j}^{n} \dot{q}_{j}(t) \int_{L-v_{x} t}^{L} c \varphi_{j}(\tilde{x}) d \tilde{x} \\
& \quad+k v_{x} \alpha(t) t+\sum_{j}^{n} q_{j}(t) \int_{L-v_{x} t}^{L} k \varphi_{j}(\tilde{x}) d \tilde{x}=F_{y}+f_{2}
\end{aligned}
$$

with

$$
\begin{aligned}
f_{1 i}= & \int_{0}^{t} k \alpha\left(t_{1}\right) \varphi_{i}\left(v_{x} t_{1}+L-v_{x} t\right) v_{x} d t_{1} \\
& +A \int_{0}^{t} k \sum_{j}^{n} \varphi_{j}(L) q_{j}\left(t_{1}\right) \varphi_{i}\left(v_{x} t_{1}+L-v_{x} t\right) v_{x} d t_{1} \\
= & \int_{0}^{t} k Y\left(t_{1}\right) \varphi_{i}\left(v_{x} t_{1}+L-v_{x} t\right) v_{x} d t_{1} \\
f_{2}= & \int_{0}^{t} k \alpha\left(t_{1}\right) v_{x} d t_{1}+\int_{0}^{t} k \sum_{j}^{n} \varphi_{j}(L) q_{j}\left(t_{1}\right) v_{x} d t_{1} \\
= & \int_{0}^{t} k Y\left(t_{1}\right) v_{x} d t_{1} .
\end{aligned}
$$

Here, $M_{t}$ is the total mass of the fixture and needle; $Y\left(t_{1}\right)=$ $\alpha(t)+\sum_{i=1}^{n} \varphi_{i}(L) q_{i}$ is the needle tip position at time instant $t_{1}$, which is time varying and derived from (10)-(12); and $F_{y}$ is the lateral steering force, which acts at the needle base in the $y$ direction.

These partial differential equations can be solved using the explicit Runge-Kutta $(4,5)$ formula, and the Dormand-Prince pair. A Matlab simulation program has been composed to simulate this model. With the applied needle base force $F_{y}$ serving as the input of the model, $\alpha$ and $q_{i}$ will change with time, thus causing the change of the needle tip position $Y\left(t_{1}\right)$ in the $y$-axis, which is the output of the model, as well as the tissue reaction forces.

B. Local Polynomial Approximation of Depth-Varying Mean Parameters Considering the inhomogeneous human tissue and the multiple tissue layers that the needle will penetrate through during surgery, here we 


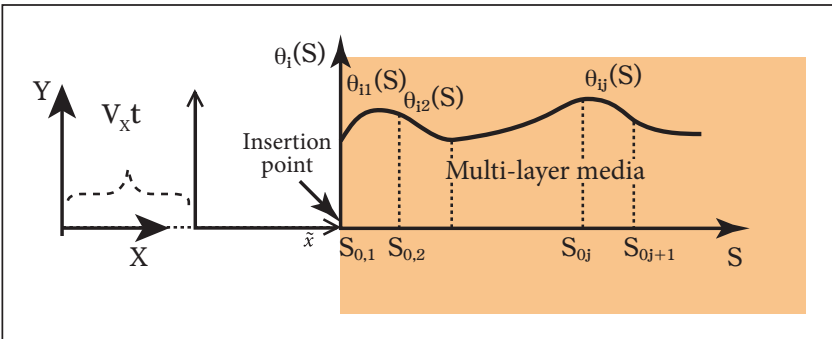

Figure 2. Local polynomial approximations of the parameter functions.

propose to use depth-varying mean parameters to calculate the spring/ damper reaction forces and use local polynomials to approximate the depth-varying mean parameters.

Assumption 1: The spring and damper coefficients are different at different depths of the tissue. At each insertion step, the spring/damper effects along the needle body that is inside the tissue can be calculated using mean spring/damper coefficients $\theta(s)=\left[\begin{array}{ll}\bar{c} & \bar{k}\end{array}\right]^{T}$. These mean coefficients will vary with each step.

This assumption takes into consideration the inhomogeneous human tissue, and at the same time, releases the computation intensity by using mean values to calculate the spring/damper forces along the needle body at each insertion step. Furthermore, the adoption of the mean values guarantees that $\theta(s)$ is continuously distributed regardless of the abrupt change of the tissue properties, e.g., pathological changes of the tissue, or multilayer insertion.

Assumption 2: The depth-varying mean parameters $\theta(s)$ can be represented by a series of local polynomial approximations in finite segments.

This can be justified using Taylor series expansion. Recall that the functions $\theta(s)$ can be expanded around certain points $s_{0}$, as shown next. Here, $\theta(s)$ is approximated by the first $p+1$ terms. The last term represents the error due to the approximation

$$
\begin{aligned}
\theta(s)= & \theta\left(s_{0}\right)+\left(s-s_{0}\right) \theta^{(1)}\left(s_{0}\right)+\cdots+\frac{\left(s-s_{0}\right)^{p}}{p !} \theta^{(p)}\left(s_{0}\right) \\
& +\int_{s_{0}}^{s} \frac{(s-\xi)^{p+1}}{(p+1) !} \theta^{(p+1)}(\xi) d \xi .
\end{aligned}
$$

From the aforesaid assumptions, we can divide the whole insertion length into several segments and adopt piecewise continuous $p$-order differentiable functions $\theta_{i j}, i=1,2, j=1,2, \ldots, n$ to represent the depth-varying mean parameter $\theta_{i}(s), i=1,2$ in each segment, as illustrated in Fig. 2. Here, the index $i$ refers to the $i$ th parameter (spring or damper coefficient), while the index $j$ refers to the $j$ th segment and $\mathrm{n}$ is the number of segments. The $S$ coordinate system is adopted for convenient representation of the parameters. So, the polynomial approximation of $\theta_{i j}$ is represented as

$$
\begin{aligned}
\theta_{i j}(s)= & a_{i j 0}\left(s_{0, j}\right)+a_{i j 1}\left(s_{0, j}\right)\left(s-s_{0, j}\right) \\
& +\cdots+a_{i j p}\left(s_{0, j}\right)\left(s-s_{0, j}\right)^{p} \\
:= & \sum_{k=0}^{p} a_{i j k}\left(s_{0, j}\right)\left(s-s_{0, j}\right)^{k} \\
:= & \varphi_{i j}^{T}\left(s, s_{0, j}\right) A_{i j}\left(s_{0, j}\right), s \in\left[s_{0, j}, s_{0, j}+l\right)
\end{aligned}
$$

where $l$ is the length of the segment and $p$ is the order of the polynomial. $s_{0, j}$ refers to the resetting depth at which the $j$ th window of the local polynomial approximation for parameter $\theta i$ begins. $s_{0, j}$ is given by the sequence $s_{0}=\left\{s_{0, j}\right\}, j=1, \ldots, n$ and $s_{0,(j+1)}-s_{0, j}=l . a_{i j k}\left(s_{0, j}\right)=(1 / k !) \theta_{i j}^{(k)}\left(s_{0, j}\right), k=0$, $\ldots, p$, where $\theta_{i j}^{(k)}\left(s_{0, j}\right)$ is the $k$ th depth derivative evaluated at $s=s_{0, j} . A_{i j}\left(s_{0, j}\right):=\left[a_{i j 0}\left(s_{0, j}\right), a_{i j 1}\left(s_{0, j}\right), \ldots, a_{i j p}\left(s_{0, j}\right)\right]^{T}$ is the unknown constant vector and $\varphi_{i j}^{T}\left(s, s_{0, j}\right):=[1,(s-$ $\left.\left.s_{0, j}\right), \ldots\left(s-s_{0, j}\right)^{p}\right]$ is a column vector. Notice that $A_{i j}\left(s_{0, j}\right)$ is constant only within each segment $\left[s_{0, j}, s_{0, j+1}\right)$, and in general, differs from one segment to another for the inhomogeneous tissue.

Therefore, it is possible to use (16) to approximate $\theta_{i j}(s)$ more precisely by choosing either a higher order polynomial, that is, $p$ large, or a smaller segment $l$, or both. If we partition the whole insertion length into segments with the length of each segment equal to $l$, then the depth-varying function $\theta_{i}(s)$ can be approximated by a number of polynomials $\theta_{i j}(s)$ located in each segment with constant coefficients $a_{i j k}$, as shown in (16).

\section{Online Parameter Estimator Design}

The discretized needle steering model is considered here. The needle steering force model can be reorganized as

$$
M \ddot{x}+c \Pi \dot{x}+\left(k \Pi+\Pi_{1}\right) x=u-k H
$$

where

$$
\begin{aligned}
x & =\left(\begin{array}{l}
\alpha \\
q
\end{array}\right), M=\left(\begin{array}{cc}
M_{t} & 0 \\
0 & 1
\end{array}\right), u=\left(\begin{array}{l}
1 \\
\beta
\end{array}\right) F_{y} \\
\Pi_{1} & =\left(\begin{array}{cc}
0 & 0 \\
0 & \omega^{2}
\end{array}\right), \Pi=\left(\begin{array}{cc}
v_{x} t & \int_{L-V_{x} t}^{L} \varphi(\tilde{x}) d \tilde{x} \\
\int_{L-V_{x} t}^{L} \varphi(\tilde{x}) d \tilde{x} & \int_{L-V_{x} t}^{L} \varphi^{2}(\tilde{x}) d \tilde{x}
\end{array}\right) \\
H & =\left(\begin{array}{c}
\int_{0}^{t} y_{1}\left(t_{1}\right) v_{x} d t_{1} \\
\int_{0}^{t} \varphi_{1}\left(v_{x} t_{1}+L-v_{x} t\right) y_{1}\left(t_{1}\right) v_{x} d t_{1}
\end{array}\right) .
\end{aligned}
$$

The measured output

$$
Y(t)=\left(\begin{array}{l}
y_{1} \\
y_{2}
\end{array}\right)=\underbrace{\left(\begin{array}{ll}
1 & \varphi(L) \\
1 & \varphi(0)
\end{array}\right)}_{B} x=B x .
$$

Here, $y_{1}$ refers to the needle tip trajectory, while $y_{2}$ is the needle base trajectory that is measured to facilitate the computation of the system state $x$.

After some algebraic manipulation, we can get

$$
Z_{k}=\Phi_{k-1}^{T} \bar{\theta}
$$

where $Z_{k}=Y_{k}, \Phi_{k-1}^{T}=\left[-T B M^{-1} \Pi_{k-2} B^{-1}\left(Y_{k-1}-Y_{k-2}\right)\right.$ $-T^{2} B M^{-1}\left(\Pi_{k-2} B^{-1} Y_{k-2}+H_{k-2}\right) \quad 2 Y_{k-1}-Y_{k-2}+$ $\left.T^{2} B M^{-1} u_{k-2}-B M^{-1} \Pi_{1 k} B^{1} Y_{k-2}\right]$, and $\bar{\theta}=\left[\begin{array}{lll}\bar{c} & \bar{k} & 1\end{array}\right]^{T} \in$ $R^{m}$ is the unknown depth-varying mean parameter vector with an additional constant 1 . 
Substituting the polynomial approximations for the depth-varying mean parameters, (19) can be represented as

$$
\begin{aligned}
Z_{k} & =\Phi_{k-1}^{T} \bar{\theta}=\underbrace{\Phi_{k-1}^{T}\left[\begin{array}{cc}
\varphi^{T}\left(s, s_{0, j}\right) & \\
\varphi^{T}\left(s, s_{0, j}\right) & \\
1
\end{array}\right]}_{\mathrm{X}_{k-1}^{T}} \underbrace{\left[\begin{array}{c}
A_{1} \\
A_{2} \\
1
\end{array}\right]}_{\vartheta} \\
& :=\mathrm{X}_{k-1}^{T} \vartheta s .
\end{aligned}
$$

Here, for simplicity, we select the same-order polynomials for the two parameters.

The transformation between the $s$ domain and $\tilde{x}$ domain is given by

$$
\left\{\begin{array}{l}
\tilde{x}=s+L-\dot{s} t \\
\dot{s}=v_{x}
\end{array}\right.
$$

under the same assumption that the needle is inserted at constant velocity.

$$
t_{0}:=\left\{t_{0, j}=\frac{s_{0, j}}{v_{x}}\right\}, \quad j=1,2, \ldots
$$

To facilitate the computation of the dynamic equation, the dataset $s_{0}=$ $\left\{s_{0, j}\right\}, j=1,2, \ldots$ can be converted to the time domain using

In discretized form

$$
k_{0, j}=\left\{t_{0, j} / T\right\}, \quad j=1,2, \ldots, n .
$$

Based on this approach, the modified least-square estimation with covariance resetting and forgetting factor is adopted to estimate the coefficients that take the form

$$
\begin{aligned}
\hat{\vartheta}_{k} & =\hat{\vartheta}_{k-1}+K_{k}\left(Z_{k}-\mathrm{X}_{k-1}^{T} \hat{\vartheta}_{k-1}\right) \\
K_{k} & =P_{k-1} X_{k-1}\left[\lambda I+\mathrm{X}_{k-1}^{T} P_{k-1} X_{k-1}\right]^{-1} \\
P_{k} & =\left[P_{k-1}-K_{k} \mathrm{X}_{k-1}^{T} P_{k-1}\right] / \lambda
\end{aligned}
$$

where $\hat{\vartheta}$ is the estimated parameter vector, $Z$ is the measurement, $\lambda$ is the forgetting factor, $P$ is the covariance matrix, and $\mathrm{K}$ is the gain.

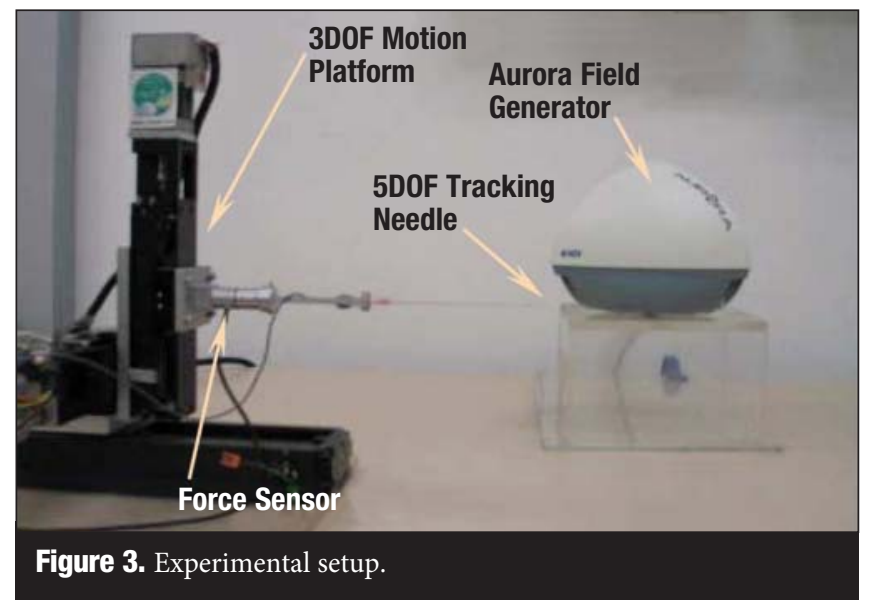

\section{Lateral Steering Force Model Validation}

1) Material and Method:

To validate the effectiveness of the proposed steering model, a physical experiment has been carried out. The experimental setup, shown in Fig. 3, is used to carry out the experiment. The 3-DOF motion platform drives the needle into the phantom/animal organ following some predesigned trajectory. A 6-DOF force/torque $(F / T)$ sensor is mounted at the needle base to measure the needle base force. The needle adopted here is a 5-DOF MagTrax needle probe. It is a 130-mm-long needle and has a sensor located at the stylet's proximal symmetric tip. This needle tip movement in the 5-DOF, except rotation about the needle axis, can be observed in real time via an electromagnetic system called Aurora.

An "active" way of validating the proposed model by steering the needle tip to a defined position is infeasible now, since it will require a steering strategy, which is our future task. Instead, a "passive" way of validation is adopted to show that the model could accurately predict the needle tip trajectory when giving some inputs - needle-based lateral forces. The detailed validation procedure is described as follows.

The needle is first driven into the prepared phantom by the 3-DOF platform following some predetermined trajectories with various insertion speeds. The needle tip/base positions and corresponding needle base force data are collected during the procedure. These collected datasets are first passed through a designed filter to remove the measurement noises and smooth the data. After that, the filled datasets go through the online parameter estimator to estimate the depth-varying mean parameters. At the same time, the model is simulated using the online estimated parameters and the collected dataset to predict the output, the needle tip position. The output is then regulated using the collected output data instead of the simulated ones during the simulation. This regulation method can prevent the simulator from accumulating estimation errors, which will gradually lead to the divergence of the estimation. At last, the simulated outputs and the needle tip position data are compared with the measured positions during experiments.

\section{2) Preliminary Experiments in Tissue-Like Phantoms:}

Phantoms made of different gelatin/water ratios were first adopted to simulate the soft tissue, for it is easy to obtain and the properties are easy to control and replicate. The needle was driven into the phantom for $8 \mathrm{~cm}$ in the $x$-direction and $2 \mathrm{~cm}$ in the $y$-direction. The insertion speed was set to be 8,4 , and $2 \mathrm{~mm} / \mathrm{s}$, respectively. The lateral speed was chosen accordingly in order to keep the movements in $x$ and $y$ to start and stop simultaneously.

Fifth-order polynomials were chosen to represent the spring and damper coefficients. The initial coefficients were set to be $\left[3 \times 10^{5} \times\right.$ ones $(6,1) ; 2 \times 10^{6} \times$ ones $\left.(6,1) ; 1\right]$. The initial covariance matrix was set to be $\left[10^{16} \times\right.$ eye $(13,12)$ zeros $\left.(13,1)\right]$. The forgetting factor was selected to be 0.99 . For comparison purpose, one segment was chosen first. Fig. 4 shows one typical example of the simulated output versus the measured output. The corresponding measurement errors and the reconstructed depth-varying mean spring/damper coefficients using the estimated polynomial parameters are shown in Figs. 5-7.

To improve the tracking accuracy, two segments were chosen next. The same initial settings were used as in the one-segment estimation. The simulation errors of one-segment estimation and two-segment estimation are compared and shown in Fig. 8. We can see that the overall accuracy has improved when using two segments. 
Fig. 4

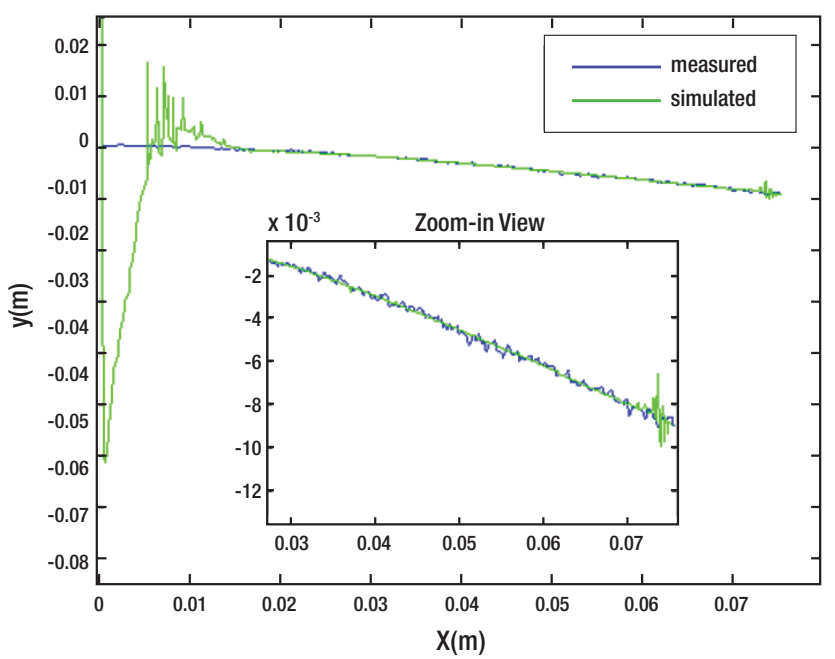

Fig. 5

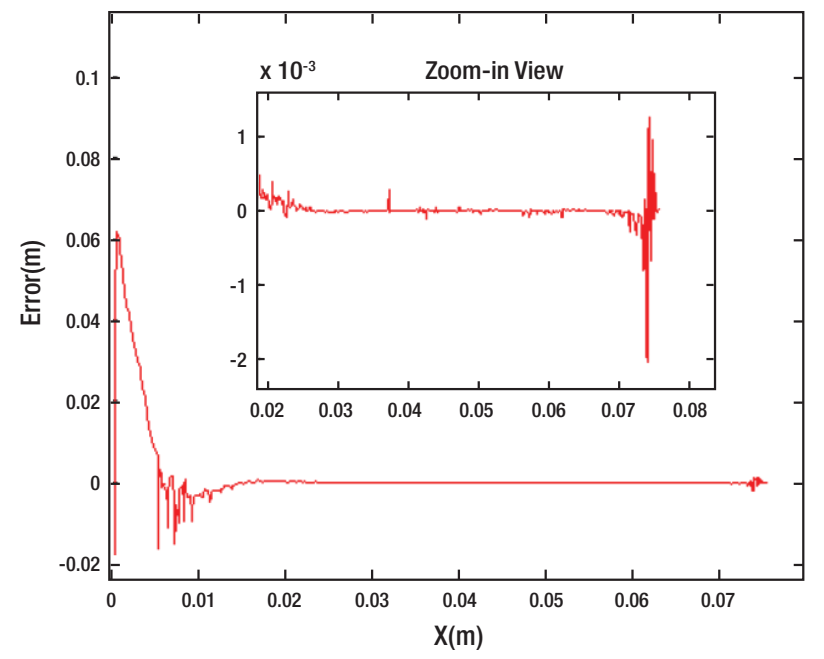

Figure 4. Simulated versus measured tip trajectories.

Figure 5. Simulation errors.

Figure 6. Estimated depth-varying spring coefficients.

Figure 7. Estimated depth-varying damper coefficients.

Figure 8. Error comparisons.

3) Discussion:

In this set of gelatin experiments, fifth-order polynomials were adopted for the spring and damper coefficients. Orders lower than fifth have shown larger estimation errors; while orders larger than fifth can give better accuracy, but no significant improvement. Dividing the whole insertion depth into more segments will improve the overall tracking accuracy, but not much improvement on the convergent rate, as can be detected in Fig. 8. The large estimation errors at the beginning were caused by poor initial estimation and the large sensor noises due to the sudden oscillation of the sensors when the needle was
Fig. 6

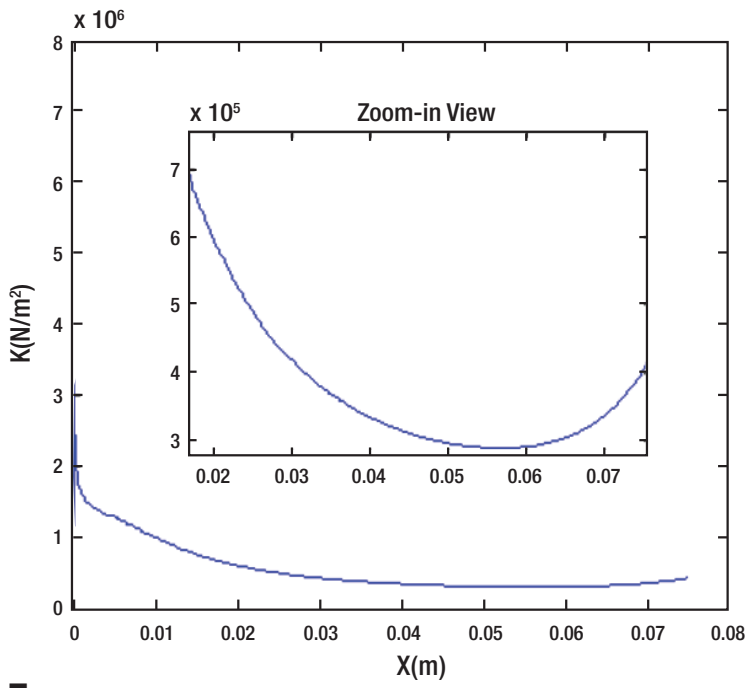

Fig. 7

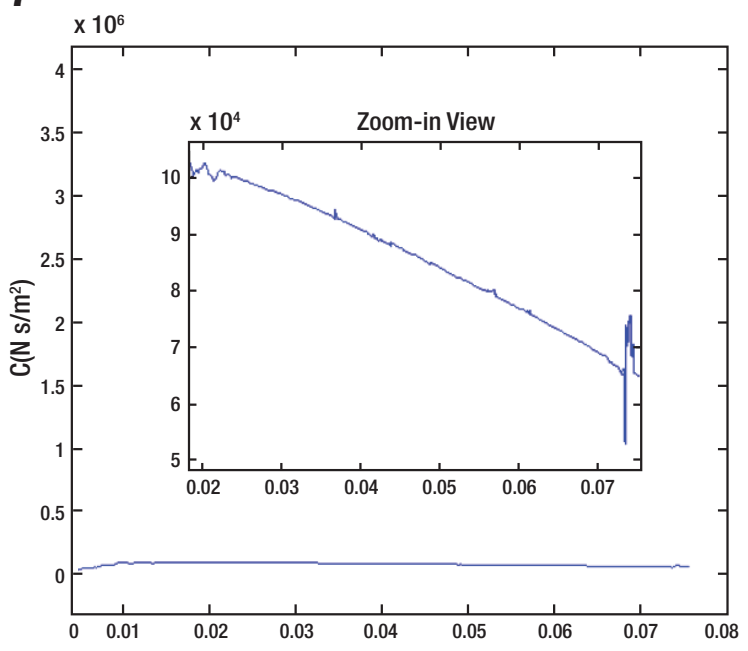

Fig. 8

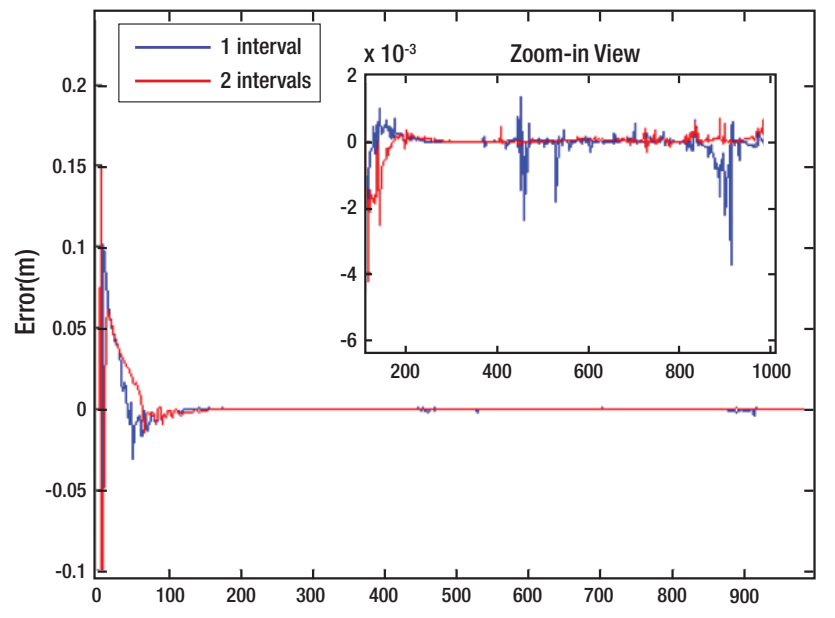


accelerated to penetrate into the phantom; the relatively large estimation errors at the end were due to the erratic sensor output when the needle was decelerated to stop. The adjustment of the initial estimation has been found to be capable of decreasing the magnitude of the initial estimation error but cannot give much improvement on the convergent rate. This will be further investigated in later experiments.

The initial covariance matrix with magnitude larger than $10^{16}$ showed a better convergent rate, but not much improvement could be achieved especially when the magnitude is larger than $10^{18}$; less than $10^{16}$ would give a poor convergent rate with a decrease in overall accuracy.

The design of the online parameter estimator guarantees that the estimated coefficients will lead to minimum errors between the measured and estimated needle positions; this means that the process and measurement errors during the procedure may be incorporated into the parameter estimates, which is allowable since our goal is to achieve accurate needle position prediction, instead of precise parameter estimation. That is why even in the homogeneous gelatin phantom, the estimated spring/damper coefficients were found to be nonuniform in depth. In addition, the estimation is specific to the chosen trajectory. Thus, even in the same medium, it is highly possible that the estimated parameters will be different when choosing different trajectories.

More experiments will be carried out in the near future to further test the robustness of the steering model, as well as finding a method to improve the convergent rate.

\section{Conclusion And Future Work}

A needle steering model is proposed in this paper. Considering the tissue inhomogeneity, depth-varying mean parameters are adopted to calculate the tissue reaction effects. Local polynomials in finite segments are adopted to approximate the unknown depth-varying mean parameters. Based on this approach, an online parameter estimator has been designed using the modified least square method with a forgetting factor. Some preliminary experiments have been carried out to verify the steering model with the online parameter estimator. Results have shown its effectiveness. More experiments will be carried out in the near future to test the robustness of the steering model and improve the convergent rate.

In the future, we will use the proposed needle steering model with an online parameter estimator to design an adaptive needle steering system that can steer the needle tip following some prescribed trajectory. The Aurora system and force sensor system can be adopted to measure the needle tip positions and needle base forces during the procedure.

\section{References}

1. J. Pouliot, R. Taschereau, C. Cot'e, J. Roy, and D. Tremblay, "Dosimetric aspects of permanent radioactive implants for the treatment of prostate cancer," Phys. Can., vol. 55, no. 2, pp. 61-68, 1999.

2. R. Taschereau, J. Roy, and J. Pouliot, "Monte Carlo simulations of prostate implants to improve dosimetry and compare planning methods," Med. Phys., vol. 26, no. 9, pp. 1952-1959, 1999

3. R. Alterovitz, J. Pouliot, R. Taschereau, I.-C. Hsu, and K. Goldberg, "Needle insertion and radioactive seed implantation in human tissues: Simulation and sensitivity analysis," in Proc. IEEE Int. Conf. Robot. Autom. Sep. 14-19, 2003, vol. 2, pp. 1793-1799.

4. R. Alterovitz, J. Pouliot, R. Taschereau, I.-C. J. Hsu, and K. Goldberg, "Sensorless planning for medical needle insertion procedures," in Proc. IEEE/RSJ Int.Conf. Intell. Robots Syst. Oct. 27-31, 2003, vol. 3, pp. 3337-3343.

5. S. P. DiMaio, "Modelling, simulation and planning of needle motion in soft tissues," Ph.D. dissertation, Univ. Br. Columbia, 2003.

6. J. Hing, A. D. Brooks, and J. P. Desai, "Reality-based estimation of needle and soft-tissue interaction for accurate haptic feedback in prostate brachytherapy simulation," presented at the Int. Symp. Robot. Res., San Francisco, CA, Oct. 2005.

7. O. Goksel, "3D needle-tissue interaction simulation for prostate brachytherapy," in Proc. 15th Annu. Can. Conf. Intell. Syst., 2005, pp. 827-834.

8. D. Glozman andM. Shoham, "Flexible needle steering and optimal trajectory planning for percutaneous therapies," in Proc. Med. Imag. Comput. Comput.-Assist. Interv., 2004, pp. 137-144.

9. R. Ebrahimi, S. Okzawa, R. Rohling, and S. Salcudean, "Hand-held steerable needle device," in Proc.Med. Imag. Comput. Comput.-Assist. Interv., 2003, vol. 2879, pp. 223-230.

10. W. Daum, “A deflectable needle assembly," Patent 5572 593, 2003.

11. M. D. O'Leary, C. Simone, T.Washio, K. Yoshinaka, and A.M. Okamura, "Robotic needle insertion: Effects of friction and needle geometry," in Proc. IEEE Int. Conf. Robot. Autom. Sep. 14-19, 2003, vol. 2, pp. 1774-1780.

12. R. J. Webster III, N. J. Cowan, G. Chirikjian, and A. M. Okamura, "Nonholonomic modeling of needle steering," Int. J. Robot. Res., vol. 25, no. 5-6, pp. 509-525, 2006.

13. Y. Zhou and G. S. Chirikjian, "Planning for noise-induced trajectory bias in nonholonomic robots with uncertainty," in Proc. IEEE Int. Conf. Robot. Autom., Apr. 26-May 1, 2004, vol. 5, pp. 4596-4601.

14. Y. Zhou and G. S. Chirikjian, "Probabilistic models of dead-reckoning error in nonholonomic mobile robots," in Proc. IEEE Int. Conf. Robot. Autom., Sep. 14-19, 2003, vol. 2, pp. 1594-1599.

15. R. Alterovitz, A. Lim, K. Goldberg, G. S. Chirikjian, and A. M. Okamura, "Steering flexible needles under Markov motion uncertainty," in Proc. IEEE/RSJ Int. Conf. Intell. Robots Syst. (IROS), Aug. 2005, pp. 120-125.

16. D. Terzopoulos and K. Waters, "Analysis and synthesis of facial image sequences using physical and anatomical models," IEEE Trans. Pattern Anal. Mach. Intell., vol. 15, no. 6, pp. 569-579, Jun. 1993.

17. F. Boux de Casson and C. Laugier, "Modelling the dynamics of a human liver for a minimally invasive surgery simulation," Lecture Notes in Computer Science, in Proc.Med. Imag. Comput. Comput.-Assist. Interv., 1999, vol. 1679, pp. 1156-1165.

18. P. F. Neumann, L. L. Sadler, and J. Gieser, "Virtual reality vitrectomy simulator," in Proc. MICCAI, 1998, vol. 1496, pp. 910-917.

19. M.-C. Lu and E. Kannatey-Asibu Jr., "Flank wear and process characteristic effect on system dynamics in turning," J. Manuf. Sci. Eng., vol. 126, no. 1, pp. 131-140, 2004.

20. F. M. C. Ching and D. Wang, "Exact solution and infinite-dimensional stability analysis of a single flexible link in collision," IEEE Trans. Robot. Autom., vol. 19, no. 6, pp. 1015-1019, Dec. 2003.

21. C. Canudas deWit, B. Siciliano, and G. Bastin, Theory of Robot Control. New York: SpringerVerlag, 1996.

22. K. Yan, W. S. Ng, Y. Yu, T. Podder, T.-I. Liu, C. W. S. Cheng, and K. V. Ling, "Needle steering modeling and analysis using unconstrained modal analysis," in Proc. 1st IEEE Int. Conf. Robot. Autom. Soc. Biomed. Robot. Biomechatronics, Italy, 2006, pp. 87-92. 